\title{
Facilitators to Immediate Post Partum Intra Uterine Contraceptive Device Uptake among Mothers Delivering in Meru Hospital
}

\author{
Consolata Kirigia*, Lucy Gitonga, Moses Mahugu Muraya \\ Chuka University, Chuka, Kenya \\ Email: *consolatakirigia@gmail.com
}

How to cite this paper: Kirigia, C., Gitonga, L. and Muraya, M.M. (2019) Facilitators to Immediate Post Partum Intra Uterine Contraceptive Device Uptake among Mothers Delivering in Meru Hospital. Open Journal of Obstetrics and Gynecology, 9, 417-441.

https://doi.org/10.4236/ojog.2019.94042

Received: February 11, 2019

Accepted: April 5, 2019

Published: April 8, 2019

Copyright $\odot 2019$ by author(s) and Scientific Research Publishing Inc. This work is licensed under the Creative Commons Attribution International License (CC BY 4.0).

http://creativecommons.org/licenses/by/4.0/

\section{Open Access}

\begin{abstract}
Family planning allows people to attain their desired number of children and determine the spacing of pregnancies. It is important to promote uptake of family planning services because it is one of the four pillars of the Safe Motherhood Initiative to reduce maternal death in developing countries. It is also enshrined in health related Sustainable Development Goals. Post Partum Intra Uterine Contraceptive Device (PPIUCD) is the oldest methods of family planning. During the ancient times, foreign bodies like stones were introduced into the woman's uterus for prevention of pregnancy. Post partum method of contraception is highly recommended because it is long acting, convenient, safe and highly effective with minimal side effects compared to the hormonal methods. Despite these advantages, the method is the lowest in use globally, regionally and locally. According to the Meru Hospital records for the year 2017, the immediate PPIUCD uptake was very low at $3.4 \%$. The broad objective of this study was to assess the factors that facilitate uptake of PPIUCD at Meru Hospital. The study site was post natal ward maternity department of Meru Hospital for a period of two months that is September and October in the year 2018. A descriptive research design was used and the study population was the mothers who were admitted in the ward during the study period. A sample size of 74 respondents was used as the representative to the target population of 289 mothers. Simple random sampling was used to get the sample for the facilitators. Data were collected using structured questionnaires and interview schedules. The data collection instruments were pretested at Embu Level five Hospital and split half reliability test technique used. Validity was ensured through review of literature from similar studies and by consulting supervisors in the department. Permission to conduct the study was sought from National Commission for Science, Technology and Innovation through Chuka University Ethics and Research Committee. De-
\end{abstract}


scriptive analysis was done to get percentages using Statistical Package for Social Science (SPSS) for windows version 23.0. Qualitative data were analyzed by grouping data into themes according to the study objectives. The analyzed data were presented in tables, charts and narratives. The findings of the study revealed that; the main client related facilitators to PPIUCD uptake were older age above 30 years, employment, tertiary level of education and knowledge on the method as a result of the providers' effort to share information. That is $70 \%$ of older aged mothers, $60 \%$ of the employed and $40 \%$ with tertiary education had the insertion. The main provider facilitator to PPIUCD uptake was timely counseling and insertion services. $20 \%$ of those who had the insertion said that timely counseling was done during antenatal period. The main method related facilitator to PPIUCD uptake was past experience and preference to alternative methods of contraception. 50\% of those who accepted the insertion had previous bad experience and side effects of other alternative methods. Some $30 \%$ of users perceived that the method was effective and convenient because once inserted no need for frequent visits to the clinic. The study concluded that clients, providers and method factors facilitated uptake of immediate PPIUCD. The study recommends putting in place strategies to improve the uptake of the method by considering the client, provider and method related facilitators.

\section{Keywords}

Facilitators, Post-Partum, Intra-Uterine, Contraceptives, Uptake

\section{Introduction}

Global stabilization of population depends on successful promotion of facilitators to uptake of contraception services [1]. The facilitators must be identified for enhancement. Family planning (FP) services are educational, comprehensive medical or social activities which enable individuals, including minors to determine freely the number and spacing of their children and to select the means by which this may be achieved [2]. Women of reproductive age who want to avoid pregnancy in developing countries are not using contraceptive methods [2]. This study sought to identify the factors that facilitate the use of contraceptives among these women of reproductive health in developing countries. Use of contraceptives is key to slowing unsustainable population growth and the resulting negative impact on the economy by having the desired number of children for every parent. The facilitators to family planning services include strong policies that strongly support family planning programs in facilitating population control [3].

Global general uptake of family planning services is at 64\%, with Africa lagging behind at 33.4\%. According to Kenya Demographic Health Survey [4], general uptake of family planning services is at $23 \%$. The global contraceptive uptake by type is that pills and injections are the most commonly used in Africa 
at $8.7 \%$ and $9.8 \%$, respectively. Uptake of Post-Partum Intra Uterine Contraceptive Device (PPIUCD) is at $3.8 \%$ while implants are at $2.3 \%$ and male condoms at $2.1 \%$. According to a study done by Pradhan et al. [5], only $2 \%$ of contraceptive users opted for PPIUCD. The study was done to determine the uptake and factors influencing the uptake of PPIUCD among the women delivering in a tertiary care hospital of Odisha, India. Most studies in Kenya have concentrated on factors determining uptake of general contraceptive methods and not specific to factors determining uptake of immediate Post Partum Intra Uterine Contraceptive Devices (PPIUCD), hence the need for this specific study of the factors facilitating uptake of this specific method.

In Nyanza western Kenya, a study found that lack of contraception awareness and low uptake is related to none visit to antenatal clinic where education on general family planning is mostly done [6]. Additionally, there were other studies on personalized contraceptive assistance and uptake of Long Acting Reversible Contraception (LARC) methods by post natal women that found knowledge on family planning methods to be a determinant to contraceptives uptake [7]. Studies have shown the most common facilitators to contraceptives uptake generally are client related such as awareness to the methods [6]. Awareness of contraceptive services through health education during focused Antenatal care was found to facilitate uptake in a study on impact of health education on unmet need of contraception among rural Egyptian women [8]. In another study, Provider related facilitators such as confidence and experience were determined [9]. More over on job training and continuous education improved midwives performance in provision of PPIUCD services resulting to increased uptake [9]. Method related facilitators such as side effects of other methods were significant in a study by Bola [10]. Side effects of other alternative methods specifically the hormonal methods prompted some participants to choice of PPIUCD [11].

Awareness of contraceptive services through health education during focused Antenatal care was found to facilitate uptake in a study on impact of health education on unmet need of contraception among rural Egyptian women [8]. A study by Wulifan et al. [12] found out that optimal contraceptive use among women of reproductive age was influenced by women who were highly knowledgeable about family planning use.

A study by Katheit and Agarwal [11] found out that acceptance of PPIUCD was higher among age group between 21 - 25 years. Uptake was also high among women who had tertiary education. This was a study on evaluation of post placental intra uterine device in terms of awareness, acceptance and expulsion in a tertiary care centre. According to a study done by Pradhan et al. [5], contraceptives use was determined by age between 25 - 29, primary and secondary level of education. The study was done to determine the uptake and factors influencing the uptake of PPIUCD among the women delivering in a tertiary care hospital of Odisha, India. In a study to investigate factors associated with male involvement in reproductive health among Bangladeshi men. Continuous education increase 
confidence and technical competence of healthcare providers on counseling and insertion of PPIUCD [13]. More over on job training improved midwives performance in provision of PPIUCD services [9]. Side effects of other methods specifically pills facilitated some participants to choice of PPIUCD [11].

Post Partum Intra Uterine Contraceptive Device is a long acting reversible contraceptive method of family planning that can be inserted safely at any time during the first 48 hours after delivery. It can also be inserted after 6 weeks postpartum and after an abortion. Immediate intra uterine device is inserted within 10 minutes to 48 hours after third stage of labor in vaginal delivery or intra-caesarian before uterine closer. After 48 hours the mother can only get the insertion after puerperium, i.e. interval IUCD. The IUCD is among the oldest methods of contraception. The modern PPIUCD is a highly effective, safe, private, long-acting, coitus independent, rapidly reversible with fewer side effects and the most cost-effective method of contraception [14]. Post Partum Intra Uterine Contraceptive Device is very convenient because it requires little attention once it is inserted. It reduces missed opportunities unlike the interval IUCD. It is very timely when the mother is highly motivated towards family planning. The motivation is related to labor process (International Federation of Gynaecology and Obstetrics) (FIGO). Despite the many advantages of PPIUCD as a method of family planning, it is not popular compared to the pills, implants and depo provera.

Meru Hospital (MH) being the county referral hospital has increased number of hospital deliveries at 5204 per year [15]. Meru county government has a functional reproductive health department. This should provide an excellent opportunity to increased uptake of PPIUCD services considering the earlier mentioned advantages. Presence of this excellent opportunity with low uptake of the services warranted this study. An uptake of as low as 3.4\% was reported in Meru Hospital, out of target of more than 50\%. The PPIUCD uptake was not well documented and there was little information on the factors that hinders or promotes its uptake. According to a study done by Pradhan et al. [5], only $2 \%$ of contraceptive users opted for PPIUCD. This research sought to determine this low uptake. Moreover, according to literature review, studies on the use of immediate PPIUCD were inadequate in Kenya. Despite the immediate PPIUCD having several benefits related to its excellent timing to avoid missed opportunities, its uptake is low prompting need for investigation. Its timely administration prevents unwanted pregnancies due to early resumption of sexual activities and unpredictable ovulation after delivery. The main objective of the study was to assess facilitators to immediate Post Partum Intra Uterine Contraceptive Device uptake among mothers delivering in Meru Hospital. The specific objectives are;

1) To determine client related facilitators to immediate Post Partum Intra Uterine Contraceptive Device uptake among mothers delivering in Meru Hospital.

2) To establish provider related facilitators to immediate Post Partum Intra Uterine Contraceptive Device uptake among mothers delivering in Meru Hos- 
pital.

3) To analyze method related facilitators to immediate Post Partum Intra Uterine Contraceptive Device uptake among mothers delivering in Meru Hospital.

This study resulted to general contribution of valuable information to institutions, to the body of knowledge, to the society and the individual researcher. This study hopes to establish an understanding on the facilitators of immediate post partum intra uterine contraceptive device uptake among mothers delivering in Meru Hospital. Findings of this research will inform Meru Hospital management on the factors influencing uptake of PPIUCD in their facility. This will help to improve their way of service delivery in order to increase the uptake. The findings can be generalized to inform policy and decision makers in the department of reproductive health on decisions to improved family planning service delivery. The study may provide information to students and lecturers in nursing and reproductive health academic institutions on factors that influence uptake of family planning services. The information derived from the study may be used to create awareness in the community to improve acceptability and uptake of the method. This will reduce the economic burden resulting from preventable morbidity and mortality rates that occur due abortions of unwanted pregnancies. Once facilitating factors to the uptake are known, appropriate measures by respective entities to improvement will be put in place. The ideas presented in this research may be used by future researchers as reference in conducting related studies.

There was some weakness in the research process that could not be controlled by the researcher. Some respondents hesitated on giving consent to the study but this was overcome by an explanation that the information given will be confidential and that the research was for academic purposes.

\section{Methodology}

\subsection{Study Site}

This is physical, social and cultural site in which the researcher conducted the study. This study was carried out in postnatal ward, maternity department of Meru Hospital for a period of two months. The hospital is the main county referral hospital located in the urban part of the county at the outskirts of Meru town. Patients were referred from rural health facilities within the county for specialized services like obstetric and gynecological services and for attention by senior midwives among others. $\mathrm{MH}$ is a teaching hospital for students from various medical schools within the Mount Kenya region.

\subsection{Study Design}

A descriptive research design was used to determine answers to the study questions at $\mathrm{MH}$ in the months of September and October 2018. This was a social science research therefore needed descriptive answers to the study questions. The purpose of this social science research was to explore, describe and explain 
the phenomenon in question.

\subsection{Population}

Population is a group of individuals with similar characteristics of interest that is post natal mothers in this particular research. From the hospital records there were 5204 deliveries annually. This translated to an average of 14 deliveries daily. According to Kenya reproductive health care bill [16], reproductive age starts from 15 - 49 years and have the right to consent willingly for their reproductive health services including minors. A Population of 289 delivering mothers who were admitted in maternity post-natal ward during the period of study in the months of June and July 2018 was targeted.

\subsection{Sampling Procedure}

A sample is a smaller group of individuals which was the representative of the whole population. Sampling is the process that the researcher used in selecting a number of individuals for the study in such a way that the individuals selected represented the larger group from which they were selected. A sample size of 74 respondents was used as a representative to the target population of 289 mothers. Respondents selection was done through simple random sampling procedure. Simple random sampling was done in such a sampling technique where every individual in the population had an equal chance of being selected. The sample size was derived according to Nassiuma [17]. According to Nassiuma [17] coefficient of variation of at most $30 \%$ is acceptable in most surveys. In this study a coefficient of variation of $20 \%$ was used and a standard error of 0.02 .

Nassiuma [17] gives the formula as follows;

$$
\text { Sample size } n=\frac{N C^{2}}{C^{2}+(N-1) e^{2}}
$$

where, $n=$ sample size;

$N=$ population from which sample is obtained, 289;

$C=$ coefficient of variance, $20 \%$;

$e=$ standard error, 0.02 ;

$289(0.2)^{2}$

$0.2^{2}+(289-1) 0.02^{2}$

$n=74$.

\subsection{Inclusion and Exclusion}

Inclusion criteria depended on the mandatory characteristics that the subjects needed to have if they were to be included in the study. In this study the inclusion criteria was the mothers willing to participate in the study. Those who had the insertion and willing to participate were included to determine uptake facilitators. Exclusion criteria were those characteristics that disqualified the subjects from inclusion in the study. In this study the exclusion criteria was those mothers who were not willing to participate in the study. 


\subsection{Data Collection Procedure}

It is a process of gathering information on variables of interest in an established systematic fashion that enables the researcher to answer questions for outcome evaluation. This study was carried out in postnatal ward, maternity department of $\mathrm{MH}$ for a period of two months. The Subjects consented before the interview and before filling in the questionnaires with the assistance of the researcher where necessary. The consent followed a detailed explanation on the nature and purpose of the study. Data collection was done in a private side room or a screened bedside depending on the client's general condition. Data were collected using structured questionnaires as the primary tools and interview schedules to gather qualitative and quantitative data. The clients filled in questionnaires first then followed by a face to face interview by the researcher to gather more information on the qualitative data.

\subsection{Data Collection Instruments}

An instrument is a device used to measure the concept of interest in research. The researcher ensured that these were ideal measuring instruments which resulted in measures that were relevant, accurate, objective, sensitive and efficient. The researcher ensured that the instruments were able to collect accurate and reliable data whereby validity and reliability tests were conducted on the instruments. Data collection was carried out using structured questionnaires as the primary tools and interview schedules to gather qualitative and quantitative data. The questionnaires were divided into sections. Section A gathered the information on social demographics, section $\mathrm{B}$ reproductive characteristics and $\mathrm{C}$ explored on other factors i.e. client, provider and method related barriers and facilitators respectively. Interview schedules had one section exploring on clients general perceptions regarding PPIUCD services. These formed the effective means of measuring the barriers and facilitators of PPIUCD method of family planning. Questionnaires were analyzed using Statistical Package for Social Science (SPSS) for windows version 23.0. Interviews schedules helped the researcher to get more detailed information regarding PPIUCD uptake as a method of FP.

\subsection{Pretesting}

Pretesting was done to establish the feasibility and practicability of the whole research design. It is the process whereby the researcher tried out the research techniques and methods which she had in mind, looked to it on how well they worked in practice, and where necessary, modified plans accordingly. The data collection tools were pretested at Embu level five hospital. The facility offers same services to mothers with similar characteristics to those in MH. Pretesting was done in order to fish out wording issues and questions that were ambiguous and difficult to answer. Ten percent of the sample size was interviewed during pretesting. This also helped the researcher estimate the length of time taken to fill one questionnaire and entire data collection period. 


\subsection{Reliability and Validity}

Reliability and validity was established to determine repeatability and accuracy of data correction instruments respectively. A reliable instrument is the one that gives same results when used more than once to collect data from the sample randomly selected from the sample population [18]. This showed the extent to which the content of the instrument would be reliable by giving similar responses each time the instrument gets administered. The pilot study was carried out on $10 \%$ of the sample size in Embu level five Hospital. The researcher used split half technique to test reliability of the data collection instruments. This involved separating the instruments into two equal halves after administration. Each of the sub sets was awarded scores to establish their reliability. To establish validity, a review of literature was done to compare and contrast the instruments with research findings from similar instruments in other studies. Moreover, consultation of experts from the department to judge and determine the completeness of the instruments was done.

\subsection{Ethical Considerations}

Permission to conduct this study was obtained from National Commission for Science, Technology and Innovation through the Chuka University Ethics and Research Committee for review and approval. The main ethical issues addressed in this research proposal included privacy during data collection by use of side rooms and screens. For the purposes of confidentiality, the subjects information was not divulged to any other person unless as authorized by the subject when necessary. An informed written consent was obtained from subjects following a detailed explanation on the nature and reasons for the research. Names of subjects were kept anonymous by writing their inpatient number on the questionnaire instead of their names. The researcher conformed to the principle of voluntary consent where the respondents willingly participated in research. Unethical behavior would occur if the researcher failed to disclose the real purpose of the research fearing the subject's refusal to participate. Site approval was also obtained from $\mathrm{MH}$ administration before starting data collection.

\subsection{Data Analysis}

In order to describe the data in meaningful terms, it had to be subjected to statistical treatment. The collected data were entered and analyzed using statistical package for social science (SPSS) for windows version 23.0. Descriptive analysis was used to obtain percentages. Qualitative data were grouped into themes and analysis was done in relation to study objectives. The analyzed data were presented in tables, charts and narratives.

\section{Results and Discussion}

\subsection{Social Demographic Facilitators}

The first objective was to determine client related facilitators to PPIUCD uptake. 
The study revealed that social demographic characteristics play a role in high uptake.

\subsubsection{Age as a Facilitator}

The highest number of PPIUCD users in Figure 1 was above 30 years of age $70 \%$, while $30 \%$ were aged 20 - 30 years.

This study identified that most of the mothers who got the insertion were aged above 30 years as indicated in Figure 1. This shows that older age was a facilitating factor towards uptake of PPIUCD. This agrees to a study on factors influencing contraceptive non-use among women of advanced reproductive age in Nigeria by Bola [10] who found that socio-demographic characteristics exert more influence on non-use. There was a significant relationship between non use and young age. It also contradicts to a study by Katheit and Agarwal [11] who found out that acceptance of PPIUCD was higher among age group between 21 - 25 years. This was a study on evaluation of post placental intra uterine device in terms of awareness, acceptance and expulsion in a tertiary care centre. It contradicts to a study done by Pradhan et al. [5], who found out that PPIUCD uptake was higher among women of age group 25 - 29 year. The study was done to determine the uptake and factors influencing the uptake of PPIUCD among the women delivering in a tertiary care hospital of Odisha, India. The study findings are contrary to a study by Wulifan et al. [12] who found that optimal contraceptive use among women of reproductive age was higher in young women. This was a study on factors associated with contraceptive use among women of reproductive age in rural district of Burkina Faso.

\subsubsection{Education as a Facilitator}

Most users in Table 1 had tertiary level of education $40 \%$, secondary and primary level users were equal at $30 \%$.

According to this study most users had tertiary level of education $40 \%$ as presented in Table 1. This implies that education above secondary level contributes to increased uptake of PPIUCD. Therefore this study is similar to another study on factors influencing contraceptive non-use among women of advanced reproductive age in Nigeria by Bola [10] who found that socio-demographic characteristics

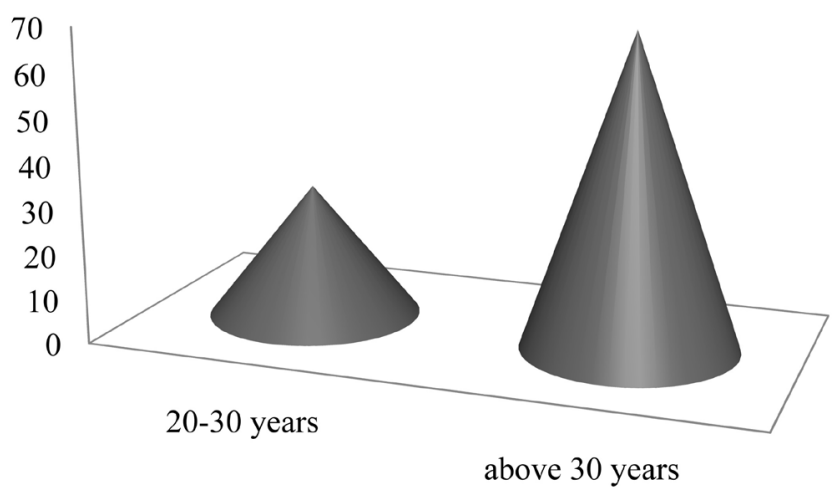

Figure 1. Age group of respondents. 
Table 1. Education level of respondents.

\begin{tabular}{cc}
\hline Education level & Percentage \\
\hline Primary & 30.0 \\
Secondary & 30.0 \\
Tertiary & 40.0 \\
\hline
\end{tabular}

exert more influence on uptake. There was a significant relationship between non use and woman's education below secondary level. It is similar to a study by Katheit and Agarwal [11] who found out that tertiary education influenced high PPIUCD uptake. This was a study on evaluation of post placental intra uterine device in terms of awareness, acceptance and expulsion in a tertiary care centre. It is similar to a study done by Pradhan et al. [5], who found out that PPIUCD uptake was higher among women who had secondary level of education. The study was done to determine the uptake and factors influencing the uptake of PPIUCD among the women delivering in a tertiary care hospital of Odisha, India. The study findings admits to a study by Wulifan et al. [12] who found that optimal contraceptive use among women of reproductive age was influenced by education status of the women. This was a study on factors associated with contraceptive use among women of reproductive age in rural district of Burkina Faso.

\subsubsection{Marital Status as a Facilitator}

Most users as indicated in Table 2 were married $80 \%$ and living with their spouses. They were influence by desire to have no more pregnancy or to have wider interval of pregnancies, otherwise the husband did not decide for them. The other $10 \%$ were single and $10 \%$ either separated or divorced.

The study indicates that most users were married, according to Table 2. During the interview session, most of the respondents were married and living with their spouses therefore they had frequent sexual intercourse. The mothers said that they preferred the method because it is effective and long acting therefore they wanted to have a wider spacing between pregnancies. This is an indication that marital status and living together with the husband influenced high uptake of PPIUCD. However they said that they did not need to consult anyone about their contraceptive choice. It disregards Rachel et al. [19] in Nyanza western Kenya in a study on overcoming barriers to family planning through integration who found that marriage was significant related to low use of FP methods. This was because married women had to consult their husbands before making a decision on family planning. The findings are similar to a study by Katheit and Agarwal [11] who found out that marital status influenced PPIUCD uptake. This was a study on evaluation of post placental intra uterine device in terms of awareness, acceptance and expulsion in a tertiary care centre. In a study on factors influencing uptake of IUCD among post partum adolescents by Melisa et al. [20] found that marital partiners had no influence on the womens' choice of 
Table 2. Marital status of respondents.

\begin{tabular}{cc}
\hline Marital Status & Percentage \\
\hline Married & 80.0 \\
Separated/Divorced & 10.0 \\
Single & 10.0 \\
Total & 100.0 \\
\hline
\end{tabular}

contraceptive use. According to Atem et al. [21], in a study on determinants of unmet need for family planning among women in Urban Cameroon: a cross sectional survey in the Biyem-Assi Health District, the major reason for non-use of contraception among women with unmet need for family planning was husband's disapproval of contraception and non-discussion of family planning within the couple. This contradicts to a study on family planning use and associated factors among pastoralist community of a far region, eastern Ethiopia by Mussie et al. [22]. The study found that three-quarters of the non-users had the influence of husband. They found that women who were married said that they needed more time to make a decision on the method of family planning to use by consulting their spouses and significant others.

\subsubsection{Employment as a Facilitator}

In Figure 2, $60 \%$ of the users had a salaried employment, $20 \%$ and $20 \%$ of the respondents were self-employed.

Figure 2 indicates that most of the users had a salaried employment. This implies that employment status influence high uptake of contraceptives. It is similar to other studies which revealed that the contraceptive use was found higher among employed women than that of unemployed women [23]. This study contradicts to a study by Katheit and Agarwal [11] who found out that employment status did not influence PPIUCD uptake. This was a study on evaluation of post placental intra uterine device in terms of awareness, acceptance and expulsion in a tertiary care centre. There were other studies on personalized contraceptive assistance and uptake of Long Acting Reversible Contraception (LARC) methods by post natal women that found employment to be a determinant to contraceptives uptake [7]. This agrees to a study in Nyanza western Kenya [24] which concluded that non use was significantly influenced by woman's occupation as a house wife. Therefore this study does not agree that low uptake was influenced by unemployment (Figure 2). The study findings are similar to a study by $\mathrm{Wu}$ lifan et al. [12] who found that optimal contraceptive use among women of reproductive age was influenced by employment status of the women. This was a study on factors associated with contraceptive use among women of reproductive age in rural district of Burkina Faso.

\subsubsection{Religion as a Facilitator}

Figure 3 shows that among the mothers using PPIUCD, 20\% were Catholics and $80 \%$ were Protestants. 




Figure 2. Employment status of respondents.

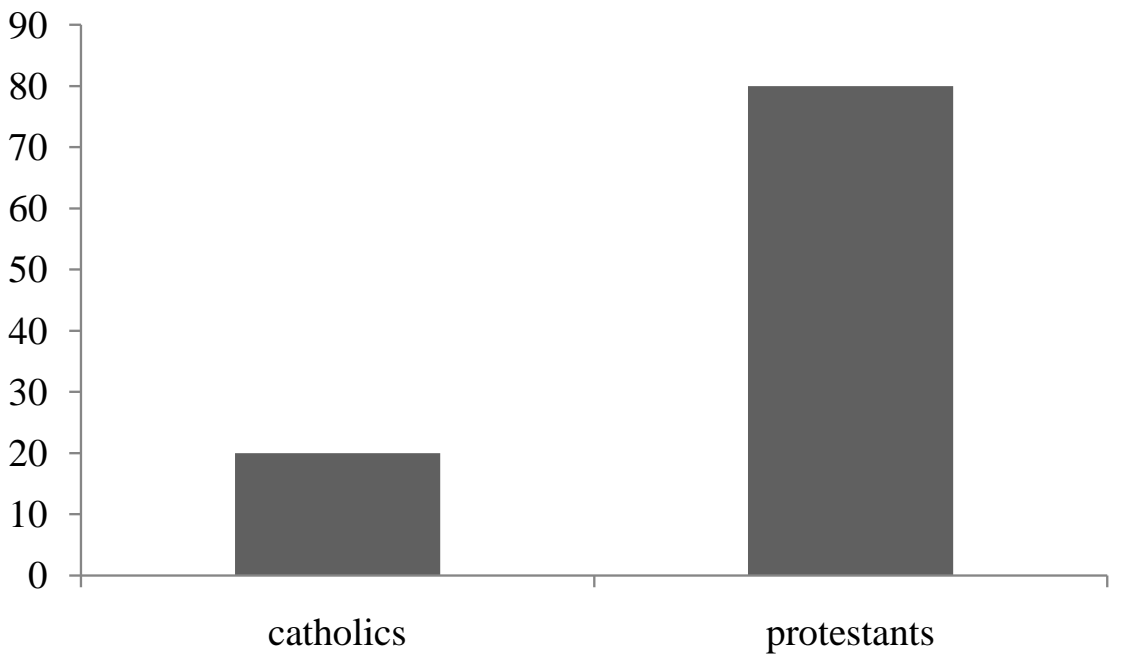

Figure 3. Religion of respondents.

The study shows that religious denominations influenced uptake. Most of them were Protestants thus high uptake because Protestants advocate for artificial methods of contraceptives. The least number were Catholics who do not advocate for artificial methods of contraception. The study findings agree to a study by Wulifan et al. [12] who found that low contraceptive use among women of reproductive age was influenced by religious beliefs of the women. This was a study on factors associated with contraceptive use among women of reproductive age in rural district of Burkina Faso. In another study on family planning use and associated factors among pastoralist community of a far region, eastern Ethiopia by Mussie et al. [22] concluded that the low coverage of family planning in the region was due to the influence of religion. This agrees to a study on access to family planning services by Muslim communities in Nepal, there was a significant association between contraceptive non use with Islamic religion [25]. The study findings agree to a study by Wulifan et al. [12] who found that low contraceptive use among women of reproductive age was influ- 
enced by religious beliefs of the women.

\subsubsection{Who Lived with the Client and Influence to PPIUCD Use}

Figure 4 indicates that $80 \%$ of respondents lived with their spouses, while $10 \%$ lived with either own parents or parents in laws. The same number $10 \%$ lived with others such as their children.

The study indicates that majority of the users were living with their spouses according to Figure 4. This is an indication that living with a marital partner influenced high uptake of PPIUCD. It disregards Rachel et al. [19] in Nyanza western Kenya in a study on overcoming barriers to family planning through integration who found that living with marital partners was significant related to low use of FP methods. This was because married women had to consult their husbands before making a decision on family planning. But in this study women made their own choice of contraceptive use to prevent or space pregnancy. The findings are similar to a study by Katheit and Agarwal [11] who found out that living together with the spouse influenced PPIUCD uptake unlike those who were separated. This was a study on evaluation of post placental intra uterine device in terms of awareness, acceptance and expulsion in a tertiary care centre. It contradicts to a study on factors influencing uptake of IUCD among post partum adolescents by Melisa et al. [20] found that living with marital partners had no influence on the women's choice of contraceptive use. A few of the mothers lived with their own mothers or their mothers in law. According to Atem et al. [21], in a study on determinants of unmet need for family planning among women in Urban Cameroon: a cross sectional survey in the Biyem-Assi Health District, the major reason for non-use of contraception among women with unmet need for family planning was due to disapproval by mothers in laws. This contradicts to a study on family planning use and associated factors among pastoralist community of a far region, eastern Ethiopia by Mussie et al. [22]. The study found that three-quarters of the non-users had the influence of their parents. The other $10 \%$ of the respondents lived with their significant others for example their children or other relatives. A study by Wulifan et al. [12] who found that optimal contraceptive use among women of reproductive age was influenced by their own choice and preference of contraceptives use. This was a

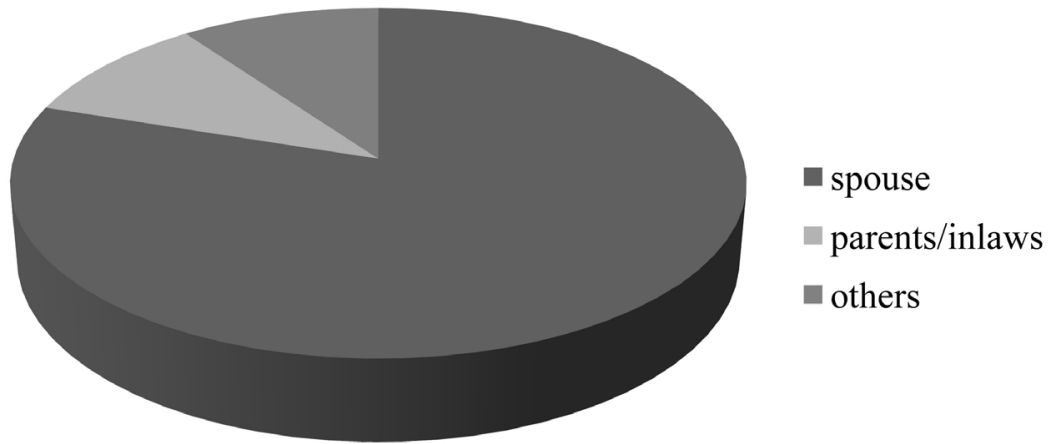

Figure 4. Who lived with the client and influence to PPIUCD use. 
study on factors associated with contraceptive use among women of reproductive age in rural district of Burkina Faso.

\subsection{Reproductive Characteristic Facilitators}

The first objective was to determine client related facilitators to PPIUCD uptake. The study revealed that reproductive characteristics play a role in high uptake.

\subsubsection{Parity as a Facilitator}

Most respondents in Table 3 were para 1 - 3 at $70 \%$ and those who were more than para 3 were at $20 \%$ while more than para 3 were at $10 \%$.

Most respondents according to the study had parity above 3 (Table 3). This shows that high parity influenced high uptake of PPIUCD. Similarly, acceptance of PPIUCD was significantly higher in multiparous women than primiparous in a study of postpartum intrauterine contraceptive device acceptance between primiparous and multiparous women in a tertiary care centre [26]. It contradicts to a study by Katheit and Agarwal [11] who found out that acceptance was high in para 2 mothers. This was a study on evaluation of post placental intra uterine device in terms of awareness, acceptance and expulsion in a tertiary care centre. There were other studies on personalized contraceptive assistance and uptake of Long Acting Reversible Contraception (LARC) methods by post natal women that found increased parity to be a determinant to high contraceptives uptake [7]. According to a study done by Pradhan et al. [5], it was found out that, PPIUCD uptake was higher among women who had more births. The study was done to determine the uptake and factors influencing the uptake of PPIUCD among the women delivering in a tertiary care hospital of Odisha, India.

\subsubsection{Pregnancy Spacing Interval and its Influence to PPIUCD Uptake}

Figure 5 discloses that $80 \%$ of the users were not willing to have another pregnancy at all while $20 \%$ were willing to have another pregnancy in more than five years.

In Figure 5, majority of the users were not willing to have another pregnancy. Most of them said during the interview session that they had achieved their desired number of children. Therefore they preferred PPIUCD because of its long acting properties, whereas $20 \%$ were willing to have another pregnancy in more than five years. This group opted for the method because they wanted a wider spacing interval between pregnancies. This means that uptake of PPIUCD depends with numbers of children one intends to have and the spacing interval.

Table 3. Parity of respondents.

\begin{tabular}{cc}
\hline Parity & Percent \\
\hline None & 10.0 \\
$1-3$ & 20.0 \\
More than 3 & 70.0 \\
Total & 100.0 \\
\hline
\end{tabular}




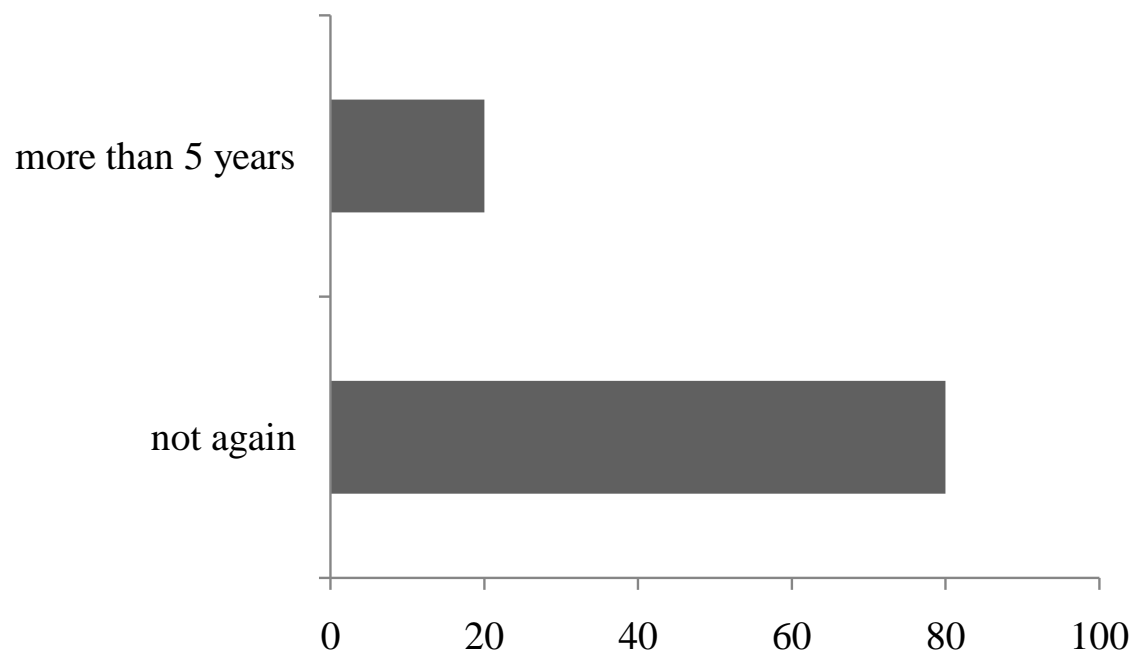

Figure 5. When the respondent intends to have another pregnancy.

This agrees to a study which found that number of living children, and child preference were found to be associated with use of contraception (Ahmed et al., 2016). Similarly, a study done by Pradhan et al. (2017), mothers who had desire for future pregnancies did not choose PPIUCD. The study was done to determine the uptake and factors influencing the uptake of PPIUCD among the women delivering in a tertiary care hospital of Odisha, India. There were other studies on personalized contraceptive assistance and uptake of Long Acting Reversible Contraception (LARC) methods by post natal women that found desire for wider pregnancy spacing interval to be a determinant to contraceptives uptake [7].

\subsection{Other Client, Provider and Method Related Facilitators}

This section discusses provider, method and other client related facilitators apart from social demographic and reproductive characteristics.

\subsubsection{When the Clients Lastly Heard about FP and Its Influence to Uptake}

Most of the users 70\% in Table 4 had heard about family planning in less than six months while others $30 \%$ did not hear about family planning in more than 6 months.

In this study most of the users as per Table 4 heard about family planning in less than six months previously. According to the interviews conducted the mothers had adequate information of family planning in general and the specific information on specific methods like PPIUCD. The mothers said that they had heard and seen advertisement about other FP during previous 6 months but the immediate PPIUCD was introduced to them by health care providers during ante natal clinics. Therefore they had specific information on PPIUCD. The study revealed that awareness of specific family planning services played a role in high uptake. This admits to a study on impact of health education on unmet 
Table 4. When the respondent lastly heard about family planning.

\begin{tabular}{cc}
\hline Lastly heard about FP & Percentage \\
\hline Less than 6 months & 70 \\
More than 6 months & 30 \\
& 100 \\
\hline
\end{tabular}

needs of contraception in urban slums of Chandigarh, India [8]. Lack of knowledge on PPIUCD was also evident in Nyanza western Kenya in a study by Pauline et al. [6]. They found that lack of awareness and low uptake was related to none visit to antenatal clinic where education was mostly done. Unlike Pauline et al. [6], this study found through interviews that the mothers visited ante natal clinics where education was done and they were taught about PPIUCD. Lack of awareness was found in a study on personalized contraceptive assistance and uptake of long acting reversible methods by post natal women [7]. Introduction of sexual education early in schools, family planning counseling services in focused antenatal clinics was found to boost public awareness of contraception [5]. The study findings support a study by Wulifan et al. [12] who found that optimal contraceptive use among women of reproductive age was influenced by women who were highly knowledgeable about family planning use. This concludes that there was adequate passing of information regarding family planning services comprehensively to the mothers. This is evidenced by adequate awareness on PPIUCD and having participation in sessions of family planning in previous 6 months.

\subsubsection{What Motivated the Respondents to Accept PPIUCD Insertion}

Table 5 gives an impression that 50\% of the users accepted PPIUCD because they had previous bad experience and side effects of other alternative methods of contraception. Some of them $30 \%$ needed a method that is long acting, safe, effective and convenient. Others $20 \%$ were informed about PPIUCD in advance during antenatal period.

There is an indication in Table 5 that majority of the users accepted PPIUCD because they had previous bad experience and side effects of other methods alternative methods especially the hormonal ones. Therefore most of them had previous experience using other contraceptives. In this case the influencing factor to the uptake is method related. Prior side effects of other methods experienced by mothers who used alternative methods specifically pills and injections influenced some participants to choice of PPIUCD [11]. Others $30 \%$ in Table 5 needed a method that was long acting, safe, effective and convenient. This was a method relate facilitator to the uptake. The modern PPIUCD is a highly effective, safe, private, long-acting, coitus independent, rapidly reversible with fewer side effects and the most cost-effective method of contraception [14]. Some $20 \%$ were informed about PPIUCD in advance during antenatal period therefore had time to ask more questions about it and to discuss with their spouses and significant 
Table 5. What motivated respondents to accept the insertion.

\begin{tabular}{cc}
\hline Motivator for insertion & Percentage \\
\hline I was informed in antenatal clinic/in in advance & 20.0 \\
Previous bad experience side effects of other methods & 50.0 \\
Need for long acting, safe, effective method & 30.0 \\
Total & 100.0 \\
\hline
\end{tabular}

others timely counseling on contraception by the providers influenced uptake. Therefore here the influence to the uptake was provider related according to Table 5. Similarly, awareness of contraceptive services through health education during focused Antenatal care was found to facilitate uptake in a study on impact of health education on unmet need of contraception among rural Egyptian women [8].

\subsubsection{Why Respondents Accepted PPIUCD Insertion without Prior Counseling}

Figure 6 demonstrates that $70 \%$ of the users had prior knowledge that PPIUCD services existed. Others $10 \%$ were given the information after delivery, $20 \%$ were counseled during their ante natal clinic visits therefore this question was not applicable for them.

Figure 6 presents that most of the users had prior knowledge from other sources that PPIUCD services existed. Therefore uptake was influenced by the client being knowledgeable about the services. Similarly, there were other studies on personalized contraceptive assistance and uptake of Long Acting Reversible Contraception (LARC) methods by post natal women which found knowledge to be a determinant to contraception uptake [7]. Studies have shown that the most common facilitators to contraception uptake generally are client related such as awareness to the contraceptive methods [6]. Awareness of contraceptive services through health education during focused Antenatal care was found to facilitate uptake in a study on impact of health education on unmet need of contraception among rural Egyptian women [8]. The study findings are also similar to a study by Wulifan et al. [12] who found that optimal contraceptive use among women of reproductive age was influenced by women were highly knowledgeable about family planning use. This was a study on factors associated with contraceptive use among women of reproductive age in rural district of Burkina Faso. A study done by Pradhan et al. [5], found out that PPIUCD uptake was higher among women who were had knowledge of the same during ante natal period. The study was done to determine the uptake and factors influencing the uptake of PPIUCD among the women delivering in a tertiary care hospital of Odisha, India. The other $10 \%$ were counseled after delivery and they did not have any doubts on competence and experience of providers. Provider related facilitators such as confidence and experience were identified in other studies [9]. More over on job training and continuous education improved midwives performance in provision of PPIUCD services [9]. 


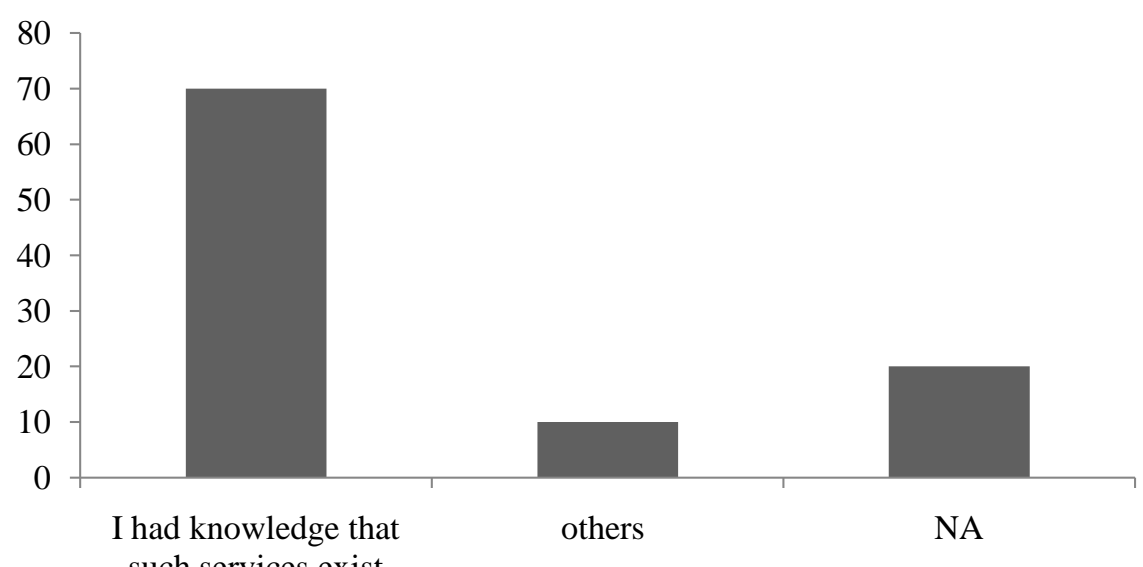

Figure 6. Motivators for PPIUCD insertion.

\subsubsection{Timing of Counseling on PPIUCD Services and Its Influence to Uptake}

Table 6 indicates that $60 \%$ of the mothers who were using PPIUCD were counseled during antenatal period. Those who were counseled after delivery were $40 \%$.

This study revealed that most of the respondents as shown in Table 6 were given counseling services on PPIUCD insertion during ante natal clinic. Those who were counseled after delivery said it was done in postnatal ward during immunization of their babies. Counseling was delayed due to shortage of staffs hence untimely services. In a study on factors influencing uptake of PPIUCD among post-partum adolescents by Melisa et al. [20] found that mothers who had consented for the method changed their mind due to delayed services. According to a study done by Pradhan et al. [5], PPIUCD uptake was higher among women who were counseled during ante natal period. The study was done to determine the uptake and factors influencing the uptake of PPIUCD among the women delivering in a tertiary care hospital of Odisha, India. In other respects, lack of enough providers or shortage of staffs contributed to delayed services in a study on midwives' views on PPIUCD [9] at nine wells hospital Dundee U.K. Task sharing as a solution to staff shortage among different healthcare providers to offer contraception services in line with their routine duties influence high uptake [13].

\subsubsection{PPIUCD User Perceptions}

In Table 7 most of the users said that they perceived PPIUCD as a method of family planning had no bad side effects. Others $30 \%$ said that it is convenient. A few of them $20 \%$ said the method is long acting.

The study results in Table 7 indicate that majority of the users said that they perceived PPIUCD as a method of family planning has no bad side effects. They did not have fears and concerns related to the side effects of the method. Side effects of other methods specifically pills and injections facilitated some participants to choice of PPIUCD [11]. Others $30 \%$ said that it is convenient because 
Table 6. When the Respondent was counseled for PPIUCD insertion.

\begin{tabular}{cc}
\hline Timing on counseling & Percentage \\
\hline During antenatal & 60 \\
After delivery & 40 \\
Total & 100.0 \\
\hline
\end{tabular}

Table 7. Facilitating perceptions on PPIUCD.

\begin{tabular}{cc}
\hline Perceptions on PPIUCD & Percentage \\
\hline Long acting method & 20.0 \\
No bad side effects & 50.0 \\
Once inserted no need for frequent visits to clinics & 30.0 \\
Total & 100.0 \\
\hline
\end{tabular}

once inserted one does not need to make those frequent visits to the clinic. While $20 \%$ of them said the method is long acting hence increased spacing of pregnancy. The modern PPIUCD is a highly effective, safe, private, long-acting, coitus independent, rapidly reversible with fewer side effects and the most cost-effective method of contraception [14]. Another study by Joseph [27] contradicts to this study. He found that that mothers perceived PPIUCD method to be associated with heavy bleeding after the insertion. This admits to a study on Factors Influencing Contraceptive Use in Sub-Saharan Africa whose findings stated that factors prohibiting contraceptive use were perceptions of method ineffectiveness in a study by [28]. There were no perceptions that the method causes medical and gynaecological problems. Having bad perceptions about FP influences uptake, such as loop can penetrate the heart, injection can cause infertility and pills can cause cancer [10]. In a different study about overcoming barriers to family planning in Nyanza, western Kenya women were concerned about side effects like heavy and prolonged menses [19].

\section{Conclusions and Recommendations}

The main client related facilitator to PPIUCD uptake identified was knowledge on the method as a result of the providers' initiative to share information. Most of the respondents said that information regarding the method was given to them. Social demographic characteristics also were client related facilitators. That is most of the older aged mothers employed and those with tertiary education had the insertion.

The main provider facilitator to PPIUCD uptake was timely counseling and insertion services. Most of those who had the insertion said that timely counseling was done during antenatal period.

The main method related facilitator to PPIUCD uptake was past experience and preference to alternative methods of contraception. Most of those who accepted the insertion had previous bad experience and side effects of other alter- 
native methods. Most of the users perceived that the method was effective and convenient because once inserted no need for frequent visits to the clinic.

The study concluded that clients, providers and method factors facilitated uptake of immediate PPIUCD. In a nut shell this study reveals that provider related facilitators such as timely counseling and provision of information and insertion services had the highest impact on PPIUCD uptake. Client and method related factors can be solved by the providers playing their role as expected.

The following recommendations were made based on the research findings.

1) From the findings of the study, having knowledge on the available services influenced increased uptake. Reproductive health department should put community based strategies in place to create awareness of PPIUCD services in the hospital, given that awareness is a key facilitator to the uptake.

2) According to the results, timely counseling resulted to acceptance of the services. The hospital administration should ensure continuous education and on job training is done to the providers. Staff motivation and staffing issues should be addressed for improvement of service delivery given that providers played a major role in facilitating the uptake.

3) From the findings of the study, properties and characteristics of family planning methods facilitated uptake of PPIUCD services. Side effects of alternative methods and their effects to the users should also be investigated.

\section{Conflicts of Interest}

No conflicts of interest declared.

\section{References}

[1] Eltomy, M., Saboula, N. and Hussein, A. (2013) Barriers Affecting Utilization of Family Planning Services among Rural Egyptian Women. Eastern Mediterranean Health Journal, 19, 400-408. https://doi.org/10.26719/2013.19.5.400

[2] World Health Organization (2018) Family Planning/Contraception.

[3] Dimoia, S. and John, P. (2016) Counting Ones Allies: The Mobilization of Demography, Population and Family Planning. East Asian Science, Technology and Society, 10, 355-376. https://doi.org/10.1215/18752160-3682679

[4] International Federation of Gynecology and Obstetrics, Kenya Obstetrics and Gynecological Society, Kenya Ministry of Health (2016) Institution Alization of Routine Post Partum Intrauterine Contraception Initiative.

[5] Pradhan, S., Kshatri, S., Behera, A. and Tripathy, M. (2017) Determinants of Uptake of Post-Partum Intra-Uterine Contraceptive Device among Women Delivering in a Tertiary Hospital, Odisha, India. International Journal of Reproduction, Contraception, Obstetrics and Gynecology, 6, 20. https://doi.org/10.18203/2320-1770.ijrcog20171968

[6] Pauline, B., Carol, M., Eva, K. and Richard, M. (2016)The Influence of Religion and Ethnicity on Family Planning. Journal of Religion \& Health, 55, 1192-1205.

[7] Katherine, B., Alison, B., Hong, L. and Keenan, E. (2013) Personalized Contraceptive Assistance and Uptake of Long Acting Reversible Contraception (LARC) Methods by Post Natal Women That Found Employment to Be a Determinant to Con- 
traceptives Uptake. Journal of Adolescent Health, 52, 59-63.

[8] Dinesh, K., Meenu, K. and Sharma, H. (2016) Impact of Health Education on Unmet Needs of Contraception in Urban Slums of Chandigarh, India. International Journal of Reproduction, Contraception, Obstetrics and Gynaecology, 80, 17-20.

[9] Cooper, M. and Lynch, P. (2015)A Survey of Midwives' Experience on PPIUCD. British Medical Journal, 93, 347-355.

[10] Bola, L. (2017) Factors Influencing Contraceptive Use and Non-Use among Women of Advanced Reproductive Age. Journal of Health, Population and Nutrition, 36, 1. https://doi.org/10.1186/s41043-016-0077-6

[11] Katheit, G. and Agarwal, J. (2013) Evaluation of Post Placental Intrauterine Device in Terms of Awareness, Acceptance and Expulsion in a Tertiary Care Centre. International Journal of Reproduction, Contraception, Obstetrics and Gynaecology, 2, 39-43. https://doi.org/10.5455/2320-1770.ijrcog20131210

[12] Wulifan, J., Mazalale, J., Jahn, A. and Hien, H. (2017) Factors Associated with Contraceptive Use among Women of Reproductive Age in Rural Districts of Burkina Faso. Journal of Health Care for the Poor and Underserved, 28, 228-247. https://doi.org/10.1353/hpu.2017.0019

[13] Jennifer, A., Kelly, G., Aletha, Y. and Cady, J. (2015) A Qualitative Study of Factors Influencing Contraceptive Choice among Adolescent School Based Health Center Patients. Journal of Pediatric and Adolescent Gynecology, 29, 3.

[14] Nipanal, H. (2015) Clinical Outcome of PPIUCD. International Journal of Reproduction, Contraception, Obstetrics and Gynaecology, 4, 765-769.

[15] Meru Teaching and Referral Hospital (2017) Records on PPIUCD Uptake.

[16] Reproductive Health Care Bill (2014) Kenya Law.

[17] Nassiuma, D. (2000) Survey Sampling. Theory \&Methods. Nairobi University Press, Nairobi.

[18] Eerl, R. (2013) Basics of Social Research. Thomson/Wadsworth, Belmont.

[19] Rachel, L., Stein, F., Sarah, J. and Maricianah, O. (2013) Overcoming Barriers to Family Planning through Integration: Perspectives of HIV Positive Men in Nyanza Province, Kenya. AIDS Research and Treatment, 2013, Article ID: 861983.

[20] Melisa, R., Summer, L., Amy, B. and Gillam, L.(2012) Factors Influencing Uptake of IUCD among Post Natal Adolescents. American Journal of Obstetrics and Gynaecology, 206, 40.e1-40.e7.

[21] Atem, B., Ajong, L. and Philip, N. (2016) Determinants of Unmet Need for Family Planning among Women in Urban Cameroon: A Cross Sectional Survey in the Biyem-Assi Health District, Yaoundé. BMC Womens' Health, 16, 4. https://doi.org/10.1186/s12905-016-0283-9

[22] Mussie, A., Hailemariam, K. and Lemma, R. (2015)Family Planning Use and Associated Factors among Pastoralist Community of Afar Region. Mekel University, Eastern Ethiopia.

[23] Ahmed, Z., Nazlu, I., Khatun, M. and Mosiur, R. (2017) Prevalence and Determinants of Contraceptive Use among Employed and Unemployed Women in Bangladesh. International Journal of MCH and AIDS, 5, 92-102.

[24] Rhoune, O., Mwende, M., Stephene, A. and Susan, K. (2015) Barriers to Modern Contraceptive Uptake among Young Women in Kenya.

[25] Alpana, S., Pragati, M., Gita, R. and Megha, R. (2016) To Assess Knowledge, Awareness and Acceptance about Contraceptive Methods during Postpartum Pe- 
riod. International Journal of Reproduction, Contraception, Obstetrics and Gynaecology, 5, 206-209.

[26] Sushila, K., Sanjaya, V., Sharma, B. and Kumari, M. (2016) A Comparative Study of Postpartum Intrauterine Contraceptive Device Acceptance between Primiparous and Multiparous Women in a Tertiary Care Centres. MLB Medical College, India. International Journal of Reproduction, Contraception, Obstetrics and Gynaecology, 5, 2304-2306.

[27] Joseph, K. (2017) Factors Associated with Contraceptive Use among Women of Reproductive Age in Rural Districts of Burkina Faso. Journal of Health Care Poor Underserved, 28, 228-247. https://doi.org/10.1353/hpu.2017.0019

[28] Sarah, R., Ucheoma, N. and Juliet, I. (2017) Factors Influencing Contraceptive Use in Sub Sahara Africa: A Systematic Review. International Quarterly of Community Health Education, 37, 79. https://doi.org/10.1177/0272684X16685254 


\section{Appendix 1}

QUESTIONNAIRE ON FACILITATORS (FOR MOTHERS WITH AN INSERTION)

Determinants of Immediate Post Partum Intra Uterine Contraceptive Device uptake among Mothers delivering at Meru Hospital

Serial no.....

IP no.

Circle the appropriate option in each question

SECTION A: social demographic characteristics

1) What is your age group?

a) Below 15 years

b) 15 - 19 years

c) 20 - 30 years

d) Above 30 years

2) What is your education level?

a) None formal education

b) Primary

c) Secondary

d) Tertiary

3) What is your marital status?

a) Married

b) Separated/divorced

c) Widowed

d) Single

4) What religious denomination do you belong?

a) Catholic

b) Protestant

c) Muslim

d) Others, specify

5) Who do you live with?

a) Spouse

b) In-laws/own parents

c) A lone

d) Others, specify

6) What is your current employment status?

a) Salaried employment

b) Unemployed

c) Self-employment

SECTION B: Reproductive characteristics

7) Year of your previous delivery if any

a) Not applicable

b) Less than two years ago

c) 2 - 5 years 
d) More than five years

8) Number of previous pregnancies (parity)

a) None

b) $1-3$

c) More than 3

9) When do you wish to have another pregnancy?
a) Not again
b) Within two years from now
c) 2 - 5 years
d) More than 5 years

SECTION C: Other factors that promote PPIUCD uptake (client, provider\& method related)

10) When did you lastly hear or read about family planning?

a) Less than 6 months ago

b) More than 6 months ago

c) Can't remember

d) Never heard

11) What motivated you to accept the insertion of PPIUCD after this delivery?

a) I was informed about the method during the antenatal clinic visits

b) My husband and relatives opinion

c) Service provider recommended it for me

d) Previous bad experience and side effects of other types of method

e) I need a long acting, safe and effective method of family planning

f) Motivated by my peers

Others, specify.

12) In case you were not given any information on PPIUCD insertion during ante natal period, why did you accept the services?

a) Had knowledge that such services exist

b) I was coerced to accept

c) I liked their services and attitude

d) I had no doubts on competence and experience of the service providers

e) Others, specify.

f) N/A

13) When were you counseled for PPIUCD insertion?

a) Not counseled

b) During antenatal period

c) On admission while in labour

d) After delivery

e) Others, specify.

14) What are your perceptions about PPIUCD as a method of family planning?

a) It is not effective

b) It is very effective 

c) Long term method
d) Does not have bad side effects
e) Convenient
f) Others

\section{Appendix 2}

\section{INTERVIEW SCHEDULE ON FACILITATORS (FOR MOTHERS WITH} AN INSERTION)

Determinants of Immediate Post Partum Intra Uterine Contraceptive Device uptake among Mothers delivering at Meru Hospital

Serial no

IP no

\section{Answer all the questions below}

1) Tell me briefly what you know about family planning generally.

2) What do you know about PPIUCD specifically and where did you get the information from?

3) Why did you prefer PPIUCD insertion done to you after this delivery?

4) What are your perceptions about PPIUCD services at Meru hospital and what others tell you about PPIUCD?

5) What method of family planning would you recommend to a friend and why?

6) What method have you been using before and why?

Comments about the corrections

1) Abstract heading and results \& discussion added

2) PPIUCD first explained in the abstract

3) Last paragraph shifted to the middle of introduction

4) Questionnaire and interview guideline provided as Appendix 1 and Appendix 2 respectively

5) Serial numbers for sub headings corrected

6) Serial numbers for figures and table corrected

7) There is no figure 8

8) I have decided to work with percentages only instead of both percentages and frequencies

9) Figures and tables have been sited well

10) Limitation of the study has been discussed

11) English mistakes corrected 